\title{
Picosecond laser treatment for Asian skin pigments: a review
}

\author{
Tin Hau Sky Wong, MBBS, MRCSEd, MScPD, MScAPS ${ }^{1,2}$ iti \\ 1 Leciel Medical Centre, Hong Kong \\ ${ }^{2}$ Medaes Medical Clinic, Hong Kong
}

\begin{abstract}
Picosecond (PS) laser is a novel dermatological laser technology that is useful in treating various cutaneous benign pigmentary disorders (BPDs), including freckles, solar lentigines, melasma, Hori macule, nevus of Ota, post-inflammatory hyperpigmentation, and tattoo. Treating Asian BPDs can be troublesome as Asian skin is typically associated with a high incidence of laser complications, such as burns, hyperpigmentation/hypopigmentation, and textural changes, which can result in various cosmetic and even psychosocial problems. This study aimed to describe the PS laser treatment for common Asian BPDs compared with traditional laser treatments. Peer-reviewed articles published from 1965 to 2018 were identified from PubMed and Google Scholar, and qualitatively reviewed with respect to the treatment of Asian BPDs. PS lasers achieved greater effectiveness and potentially fewer complications in the treatment of Asian BPDs through breakthrough mechanisms including photomechanical effects and laser-induced optical breakdown. PS laser is especially suitable and effective for treating various BPDs in Asian skin.
\end{abstract}

Keywords: freckles; Hori macule; melasma; nevus of Ota; picosecond laser; tattoo

\section{Introduction}

Asian skin is unique in that it is relatively unstable with respect to producing pigments [1]. Therefore, it is not surprising that Asian skin is commonly associated with benign pigmentary disorders (BPDs) [2]. There were 4.57 billion Asians globally in 2019 , equivalent to $59.66 \%$ of the total world population. Therefore, the chance of encountering BPDs in Asian patients is high and understanding the treatment of BPDs in Asian skin is important. Nowadays, the standard of treatment for BPDs is laser therapy. However, as Asian skin is prone to laser-induced complications, including hyperpigmentation/hypopigmentation, post-inflammatory hyperpigmentation (PIH), burns, and related textural changes, a rather gentle laser setting is recom- mended [1-3]. Worst still a gentler laser application with lower fluence may reduce the treatment efficacy. Therefore, the therapeutic margin for traditional laser treatment is narrow in Asians. Owing to technological advances, picosecond (PS) laser, a novel laser technology with less irritation and more efficacy (LIME), has been developed. In general, PS laser adopts lower fluence and causes less heat irritation to surrounding tissue by applying concentrated energy in an extremely short pulse duration. It predominantly yields photomechanical effects (PMEs) and stress destruction effects to targets $[4,5]$. It can break down the pigment particles into smaller pieces than can traditional quality-switched (QS) lasers, thereby allowing the immune system to more efficiently remove the fractured pigments. Further, it has been histologically proven that QS lasers more easily 
damage the surrounding tissue than PS lasers [6]. Therefore, PS lasers have better treatment margin and efficacy than QS lasers, and are a good therapeutic option for Asian BPDs. This review article will discuss the use of PS laser treatment and new laser wavelengths for various Asian BPDs.

\section{Literature search methods}

We searched PubMed and Google Scholar to identify relevant articles published between 1965 and 2018, using the following keywords: laser, Asian, freckles, lentigines, benign pigmentary disorders, picosecond, melasma, acquired bilateral nevus of Ota-like macules, ABNOM, Hori macule, nevus of Ota, NOO, post-inflammatory hyperpigmentation, and tattoo removal. We then ascertained which papers were distinctly relevant to the treatment of BPDs in Asian skin using PS lasers, for inclusion in our review.

\section{Freckles and solar lentigines}

Freckles and solar lentigines are well-defined macular BPDs, most commonly occurring in the convex areas of the face including the nose and maxillary regions. Traditional QS lasers yield as high as $76 \%$ improvement [7], and $>80 \%$ of cases achieve 50\% improvement [8]. However, these levels of improvement are still unsatisfactory. Further, as mentioned above, the characteristics of Asian skin make it prone to complications. The hypopigmentation and PIH rates were reported to be as high as $25 \%$ and $10 \%$, respectively [9]. Considering the LIME property alone, PS laser is a good novel treatment for such conditions. It more efficiently breaks down melanin while causing less irritation and fewer complications. It has been reported that PS laser is highly efficient against solar lentigines, yielding a treatment response rate of $93.02 \%$ with $>75 \%$ clearance with only a single treatment, whereas the PIH rate was reported to be lower at $4.65 \%$ with a demonstration of minimal disruption of the epidermo-dermal junction when compared with traditional QS lasers [6]. Newer laser wavelengths with PS technology, such as $670 \mathrm{~nm}$, allows deeper penetration and even fewer complications to the normal epidermal melanin while causing less irritation to hemoglobin and blood vessels owing to its high melanin-to-hemoglobin ratio (Fig. 1) [10]. Thus, the safety margin can be further improved with these wavelengths, resulting in even more LIME procedure.

\section{Melasma}

Melasma is a common BPD in the Asian population. It involves complex and multiple disease mechanisms, from hormonal effect to melanin incontinence and skin structural problems [9,11-14]. Therefore, a multi-tiered approach, from melanin inhibition to melanin disintegration and elimination, is required. Traditional combined treatments including chemical peeling and melanin-stabilizing agents have important roles. Laser treatment can be helpful but can also result in rebound of melasma if inappropriately used. It has been reported that irritation and erythema can be associated with hyperpigmentation [15]. In this way, traditional QS lasers, with their narrower therapeutic margin, may more easily result in rebound. Moreover, it has been reported that QS lasers can also give rise to severe mottled hypopigmentation/hyperpigmentation [16]. Therefore, a LIME laser therapy is required and PS laser is a good choice. The author uses a PS laser with pulse width of $750 \mathrm{ps, \text {wave- }}$ length of $1,064 \mathrm{~nm}$, and fluence of 0.6 to $1.2 \mathrm{~J} / \mathrm{cm}^{2}$ at $10 \mathrm{~Hz}$, for 2,000 to 4,000 counts each time at intervals of 3 to 4 weeks between 6 treatments, together with optional adjuvant treatments (including long-pulsed 1,064/532 nm laser for vascular components, chemical peels, and topical treatments). This procedure yields good improvement. An example case is shown in Fig. 2. Owing to the LIME property of PS lasers, multiple BPDs, including freckles and solar lentigines, on the background of melasma can be treated with 532/670 nm PS lasers at low fluence, with good outcomes.

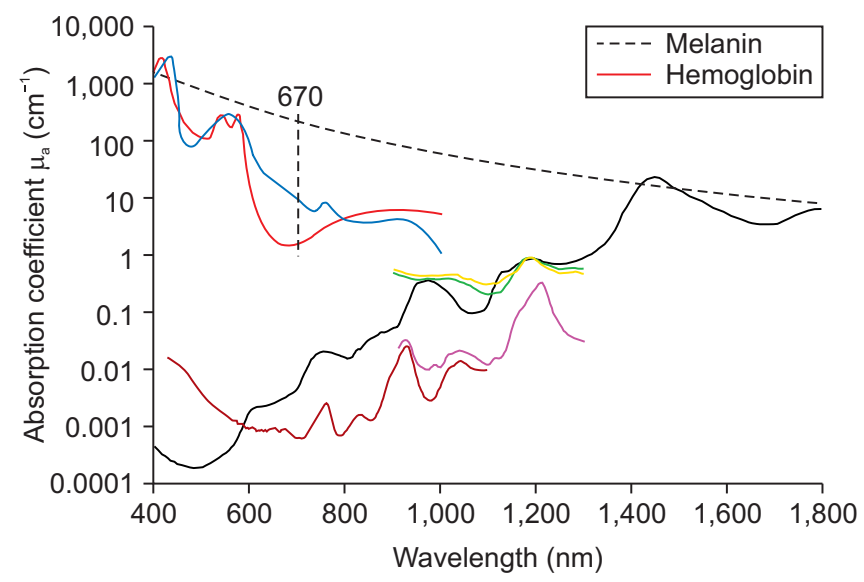

Fig. 1. The 670-nm melanin-to-hemoglobin absorption ratio is higher than contemporary wavelengths including 755 and $785 \mathrm{~nm}$. Cited from the article of Beard P (Interface Focus 2011;1:602-31) [10] with original copyright holder's permission. 


\section{Acquired bilateral nevus of Ota-like macules/ Hori macule and nevus of Ota}

Acquired bilateral nevus of Ota-like macules (ABNOM) is an acquired condition affecting up to $7.5 \%$ of dyspigmentation cases [17]. It presents as a type of bilateral bluish-brown pigmentary disorder most frequently occurring over malar areas, but may also occur over the forehead, temple, and alae of the nose. Microscopically, it is composed of a cellular component with bipolar melanocytes that are rather deep seated in the papillary and upper reticular dermis, and of larger melanosomes with increased melanosis. Historically, traditional QS lasers required high power and numerous treatments to achieve only partial improvement, while complications such as PIH and hypopigmentation rather often occurred $[18,19]$. The downtime after these procedures was long. With the use of PS lasers, much lower fluence, fewer treatments, shorter downtime, and fewer complications were encountered with satisfactory results [20]. The parameters used were $750 \mathrm{ps}$ and a spot size of 3-5 $\mathrm{mm}$. The fluence was 1.8 to $3.0 \mathrm{~J} / \mathrm{cm}^{2}$ at $1,064 \mathrm{~nm}$ with optional use of a micro-lens array (MLA) and 0.1 to $0.3 \mathrm{~J} / \mathrm{cm}^{2}$ at $532 \mathrm{~nm}$ (Fig. 3 ). The use of MLA may contribute to elimination of the cellular component of ABNOM, which previously caused pigments to be resistant and to respond inconsistently to treatment. Similar results were also concluded in a previous study [21] in which PS laser treatment yielded better clinical outcomes and fewer adverse effects than QS laser treatment.

Nevus of Ota (NOO) is a pathologically similar but congenital condition occurring in childhood and infancy, affecting up to $2.5 \%$ of the Asian population [22]. It presents as unilateral grayish hyperpigmentation on the face and sclera, usually at the trigeminal nerve distribution. Histologically, it consists of speckled elongated dendritic melanocytes with large melanin granules [23]. Therefore, problems in both melanin produc-
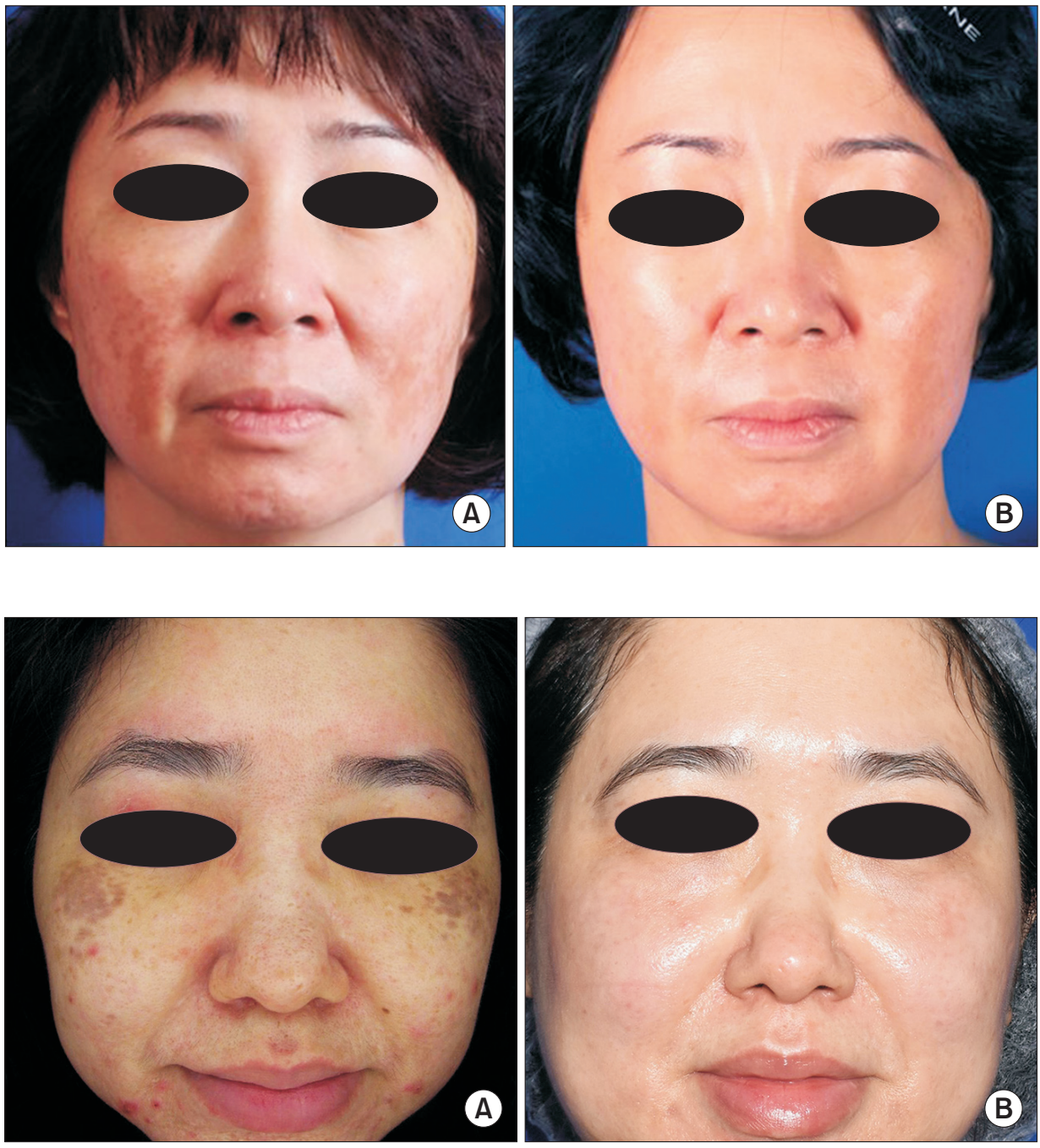

Fig. 2. A case of typical malar facial melasma with vascular components that was successfully improved with picosecond (PS) laser treatment. (A) Before treatment, (B) after 6 PS laser treatments.
Fig. 3. Clinical remission of acquired bilateral nevus of Ota-like macules (A) compared with the condition before picosecond laser treatment (B). 
tion and abnormal melanocytes exist. As the pigment occurs at an eye-catching position on the face, it can pose significant psychosocial disturbance to the patient. Therefore, treatment of the condition is important. In the past when QS lasers were used, numerous treatments with high fluence were required [24-29] and resulted in relatively high rates of complications including hypopigmentation/hyperpigmentation [30]. There have been cases of NOO that reached a plateau phase after a few treatments with QS lasers, resulting in a signature "panda sign" (darker pigment remnant in the periorbital area). This pigmentation pattern is partly because the delicate eyelid cannot be treated with the destructive high fluence that QS lasers required for efficacy. With the emergence of PS lasers, the PMEs have led to a more efficient breakdown of the pigment in a gentler way by generating less heat. The author found coincidently better results with further improvements in the plateau phase (Fig. 4) with the combined use of 532/1,064 nm and MLA PS laser. In the QS era, ruby (694 nm), alexandrite (755 nm), and neodymium-doped yttrium aluminum garnet (1,064 nm) lasers were used. Among these, the clinical outcomes of ruby and alexandrite were the most promising (Table 1) [24-29]. The contemporary wavelengths in this range for PS lasers are 670, 755 , and $785 \mathrm{~nm}$. Among these, the melanin-to-hemoglobin ratio for $670 \mathrm{~nm}$ is higher than that for 755 or $785 \mathrm{~nm}$, with a trough of hemoglobin absorption at around $670 \mathrm{~nm}$ (Fig. 1) [11]. Therefore, theoretically, it is more specific to melanin-related conditions with a smaller insult to the vascular component of the skin. It is especially important for NOO, in which melanin deposition occurs in the dermal layer and is surrounded by rich capillary networks.

\section{Post-inflammatory hyperpigmentation}

$\mathrm{PIH}$ is a complicated condition that involves a complex of pigmentary disorders composed of excessive epidermal melanin, irritated/hyperactive melanocytes, breaches of the basement membrane, melanin incontinence, abnormal dermal melanin deposition, melanophage formation, hemosiderin deposition, and a complex of inflammatory factors [31,32]. The most important consideration before addressing PIH is to avoid further irritation and re-induction of inflammation, which may give rise to secondary PIH. Therefore, a LIME regimen is required and the use of PS lasers is warranted.

\section{Tattoo}

Tattoos are common in the Asian population. Although there are no systematic epidemiological studies thus far, cultural changes have precipitated an increase in the number of individuals with tattoos in Asia, like in Western countries, particularly in the adolescent group [33]. Moreover, as eyebrow shape is a very important appearance feature for Asians, eyebrow tattoos are very common. However, a number of individuals later regret their decision to get tattooed. The reasons include a desire to dissociate from the past, unfashionable designs, or poor quality. Therefore, it is useful and important to know how to remove tattoos from Asian skin. As previously mentioned, Asian skin is laser-unfriendly; therefore, it requires PS laser procedures with LIME property. Although PS lasers show a degree of "colorblindness" when compared with QS lasers (probably owing to the PMEs), chromophore selectivity still exists. Thereby, therapeutic laser wavelengths are very important. As described
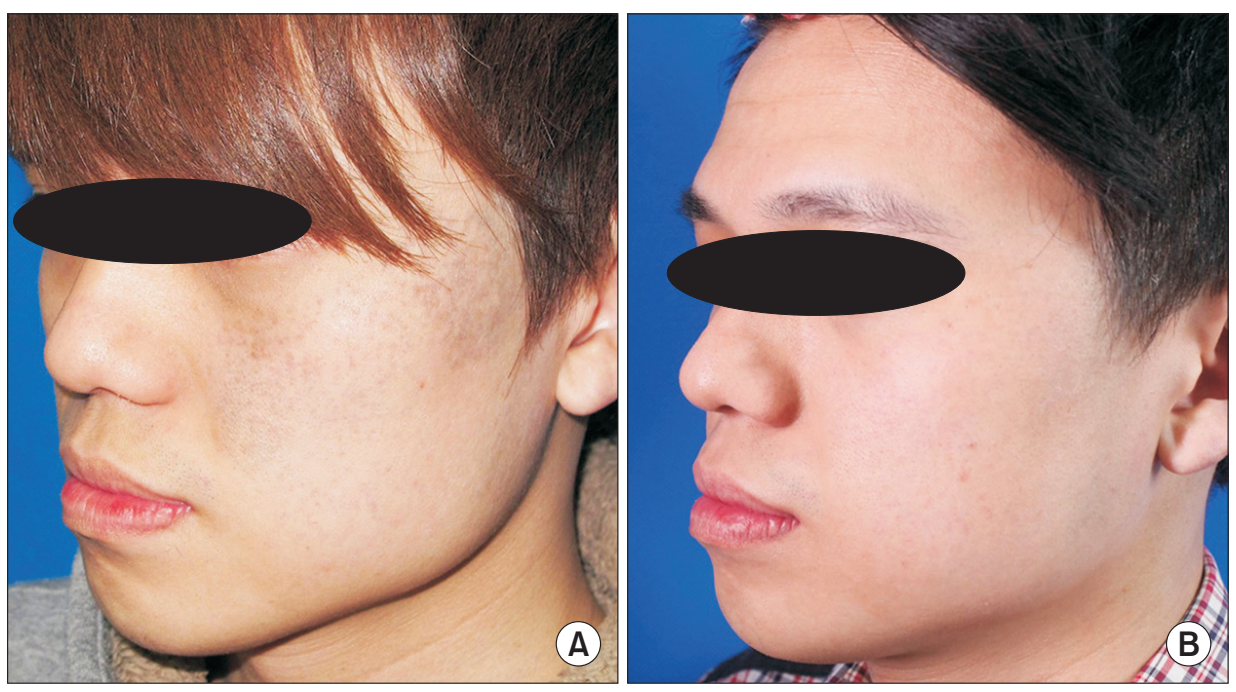

Fig. 4. (A) A 26-year-old male patient who had undergone 9 treatments with the quality-switched ruby laser, showing a plateau phase with some periorbital pigments (panda sign). Note that the patient chose to wear his hair long to cover the facial pigments. (B) The pigments were significantly reduced after 4 treatments with the picosecond laser. Note that the patient had changed his hairstyle accordingly. 


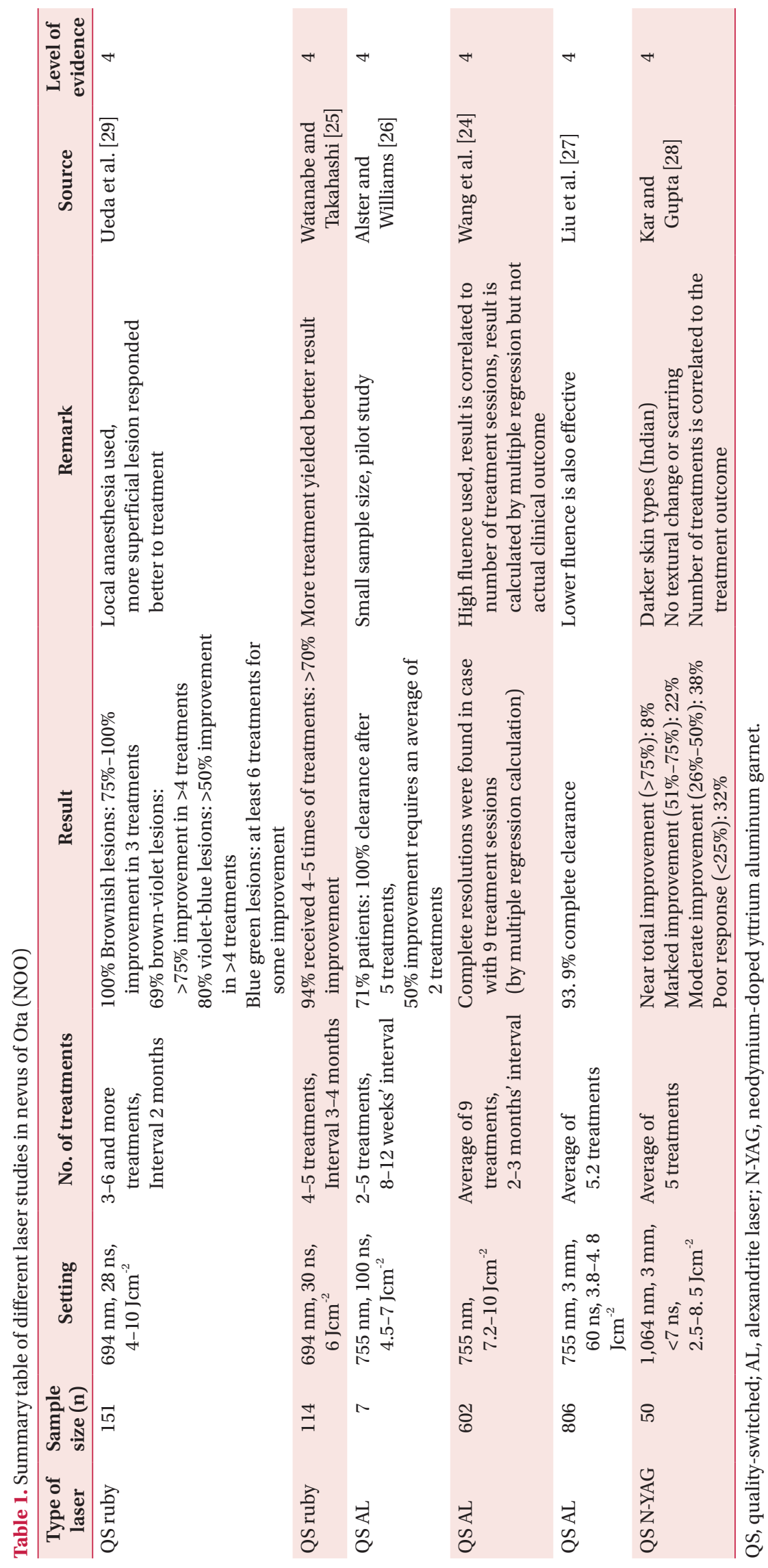


in the Introduction, the PMEs of PS laser break down the pigment particles into smaller pieces than can traditional QS laser. Therefore, recalcitrant tattoo inks can theoretically be easier to completely remove clinically (Fig. 5) or be removed to a greater degree with PS lasers (Fig. 6), which has also been demonstrated in a previous study [34]. The author has also achieved much lower rates of transient hair loss in eyebrow tattoo removal owing to the LIME property of PS lasers. PS lasers also make the removal of large tattoos less painful during treatment, with fewer complications postoperatively. They also reduce the number of treatments required to remove the pigment. In one of the author's cases, the Kirby-Desai Scale score [35] was 15 and 6 treatments were needed to achieve $>80 \%$ improvement (Fig. 7). Furthermore, although approximately $90 \%$ of the tattoo colors were red and dark blue or black, which are rather efficiently addressed with 532 and 1,064 nm wavelengths, there is an increasing utilization of new colors such as green and light blue, which are better absorbed by wavelengths of 670,755 , or 785 $\mathrm{nm}$. There is evidence that PS lasers in this wavelength range can effectively remove blue and green colors. Nonetheless, among all these wavelengths, the absorption coefficient of 670 $\mathrm{nm}$ for blue and green is the highest (Fig. 8). When compared
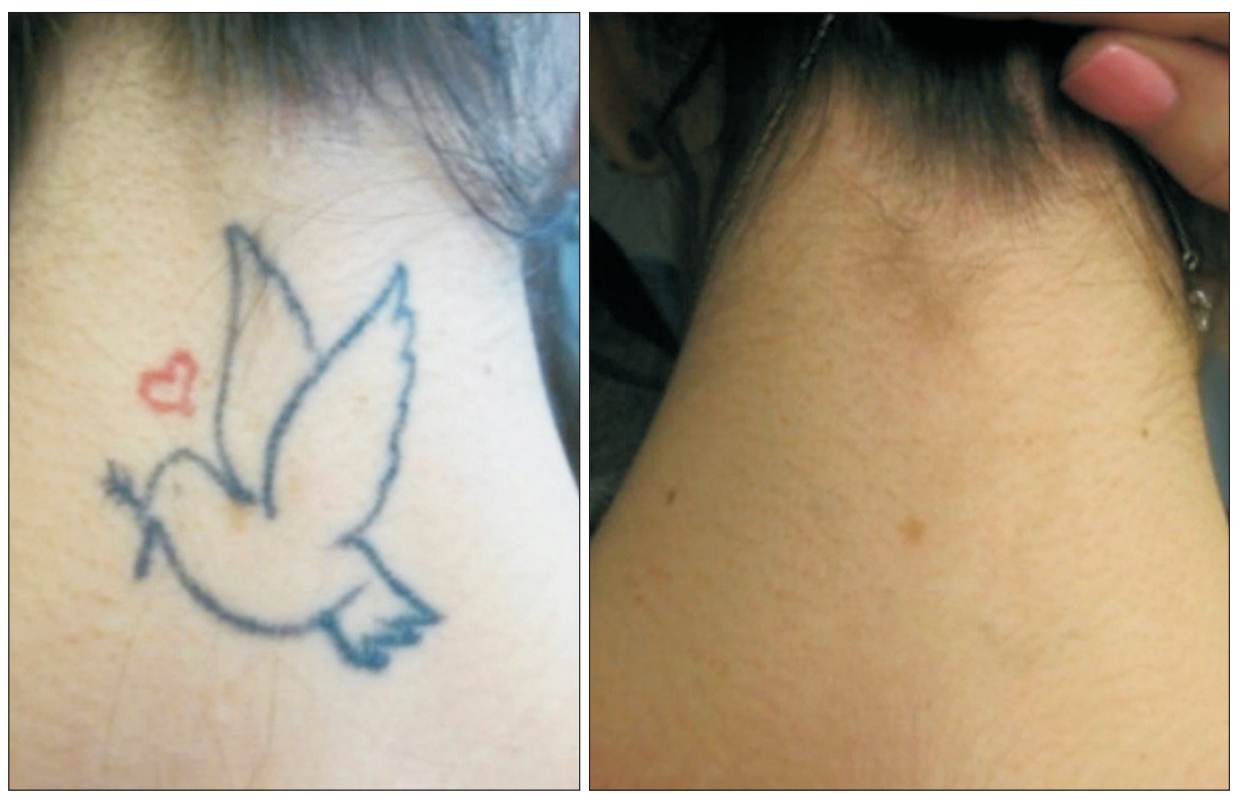

Fig. 5. Nearly complete removal of a tattoo with the picosecond laser (Courtesy of Dr Omar Ibrahimi).
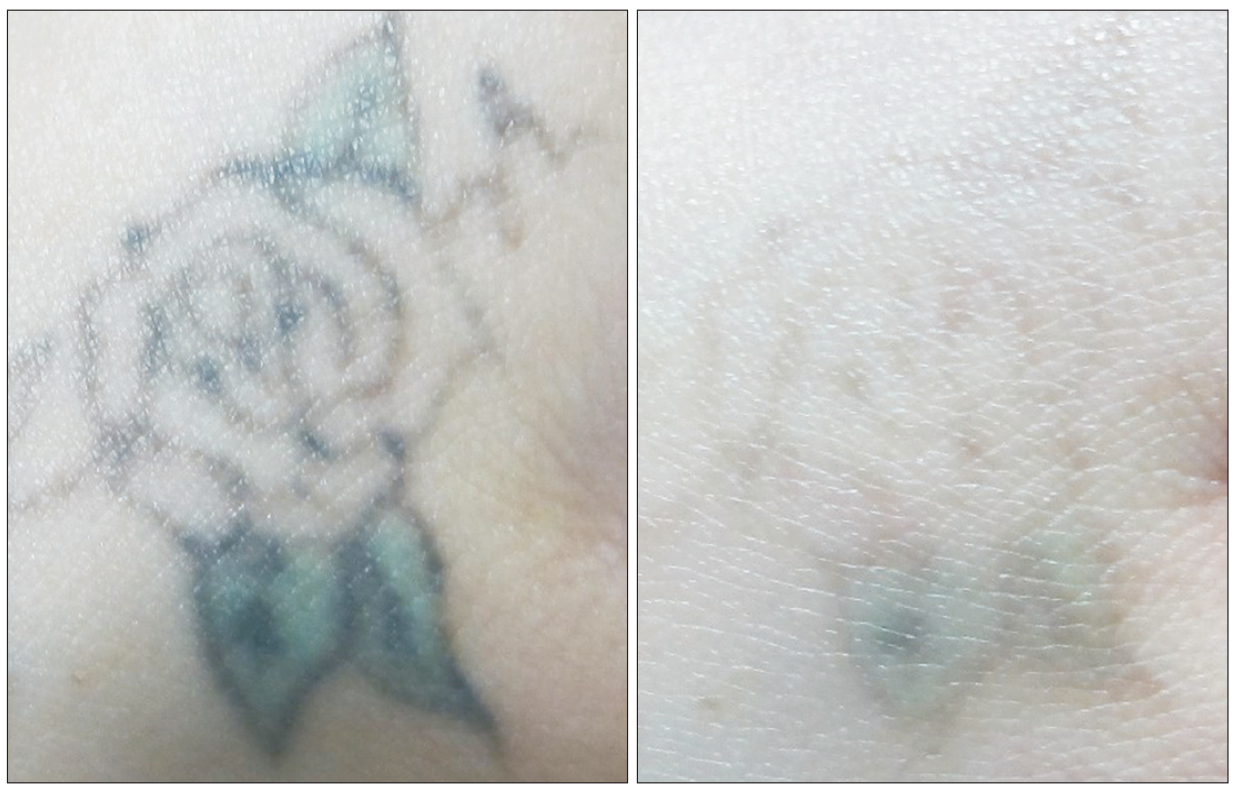

Fig. 6. Tattoo with a plateau effect from traditional quality-switched laser demonstrated further improvement after picosecond laser treatment. 

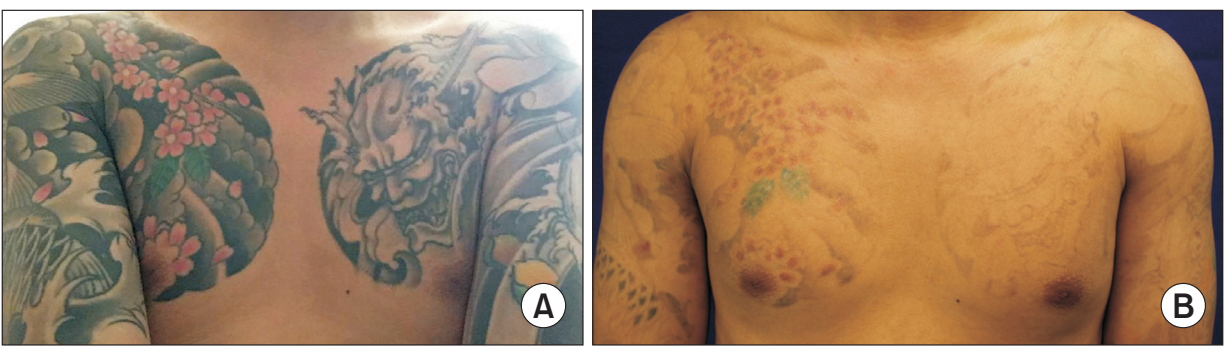

Fig. 7. Treatment of a large tattoo area. Picosecond (PS) laser treatment was effective and caused less pain to the patient during the procedure. (A) Before PS laser treatment. (B) After 6 PS laser treatments.
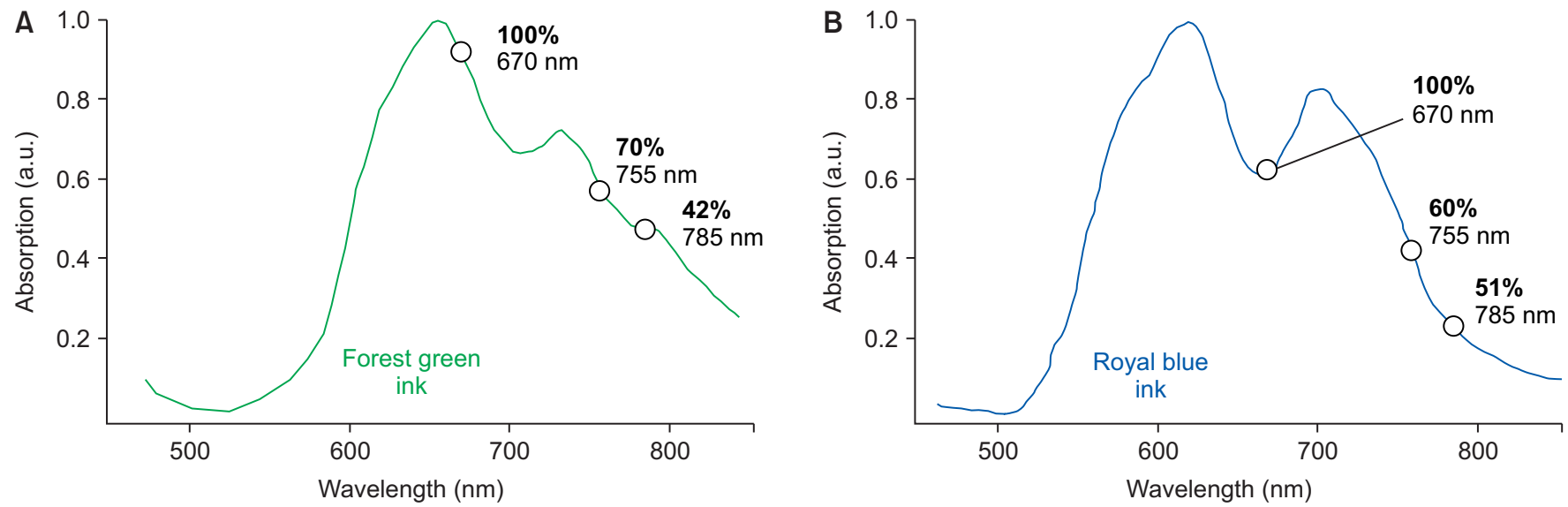

Fig. 8. Absorption spectra of green (A) and blue (B) colors with respect to laser wavelengths. a.u., arbitrary units.

with traditional QS lasers, PS lasers use lower energy, result in a shorter downtime, cause less patient discomfort, have fewer complications, and reduce the number of treatments needed. With multiple wavelengths in addition to 1,064 and $532 \mathrm{~nm}$, tattoo removal treatments can be much more effective than in the past.

\section{Conclusion}

PS lasers are a dermatological laser breakthrough. Their PMEs provide the lasers with LIME property, and together with newly developed laser wavelengths, PS lasers can tackle several Asian BPDs efficiently with improved therapeutic margins. The indications for PS lasers are increasing. Apart from various BPDs, cutaneous rejuvenations and treatments of atrophic scars have also been demonstrated to respond to the laser-induced optical breakdown effect. Multiple wavelengths provide more effective and flexible treatment of various conditions. Finally, more systematic studies on protocols of various PS laser treatments are required to demonstrate additional evidence-based benefits of these lasers and to reveal their full potential.

\section{Acknowledgments}

The author is the speaker for various academic dermatological and aesthetic conferences and Cutera Inc. for laser technologies.

\section{Conflicts of interest}

The author has nothing to disclose.

\section{References}

1. Chan HH. Effective and safe use of lasers, light sources, and radiofrequency devices in the clinical management of Asian patients with selected dermatoses. Lasers Surg Med 2005; 37:179-85.

2. Chan NP, Ho SG, Shek SY, Yeung CK, Chan HH. A case series of facial depigmentation associated with low fluence Qswitched 1,064 nm Nd:YAG laser for skin rejuvenation and melasma. Lasers Surg Med 2010;42:712-9.

3. Negishi K, Akita H, Tanaka S, Yokoyama Y, Wakamatsu S, Matsunaga K. Comparative study of treatment efficacy and the incidence of post-inflammatory hyperpigmentation with 
different degrees of irradiation using two different qualityswitched lasers for removing solar lentigines on Asian skin. J Eur Acad Dermatol Venereol 2013;27:307-12.

4. Sierra R, Mirkov M. Impact of pulse duration from nanoseconds to picoseconds on the thermal and mechanical effects during laser interaction with tattoo targets. 2013 American Society for Laser Medicine and Surgery Annual Conference;2013 Ap 3-7; Wiley Periodicals, Inc., Boston, USA. p.3-4.

5. Fabi SG, Metelitsa AI. Future directions in cutaneous laser surgery. Dermatol Clin 2014;32:61-9.

6. Negishi K, Akita H, Matsunaga Y. Prospective study of removing solar lentigines in Asians using a novel dual-wavelength and dual-pulse width picosecond laser. Lasers Surg Med 2018;50:851-8.

7. Jang KA, Chung EC, Choi JH, Sung KJ, Moon KC, Koh JK. Successful removal of freckles in Asian skin with a Q-switched alexandrite laser. Dermatol Surg 2000;26:231-4.

8. Rashid T, Hussain I, Haider M, Haroon TS. Laser therapy of freckles and lentigines with quasi-continuous, frequencydoubled, Nd:YAG (532 nm) laser in Fitzpatrick skin type IV: a 24-month follow-up. J Cosmet Laser Ther 2002;4:81-5.

9. Kang HY, Suzuki I, Lee DJ, Ha J, Reiniche P, Aubert J, et al. Transcriptional profiling shows altered expression of wnt pathway- and lipid metabolism-related genes as well as melanogenesis-related genes in melasma. J Invest Dermatol 2011;131:1692-700.

10. Beard P. Biomedical photoacoustic imaging. Interface Focus 2011;1:602-31.

11. Kim EH, Kim YC, Lee ES, Kang HY. The vascular characteristics of melasma. J Dermatol Sci 2007;46:111-6.

12. Goh CL, Dlova CN. A retrospective study on the clinical presentation and treatment outcome of melasma in a tertiary dermatological referral centre in Singapore. Singapore Med J 1999;40:455-8.

13. Wolf R, Wolf D, Tamir A, Politi Y. Melasma: a mask of stress. $\mathrm{Br}$ J Dermatol 1991;125:192-3.

14. Kurita M, Kato H, Yoshimura K. A therapeutic strategy based on histological assessment of hyperpigmented skin lesions in Asians. J Plast Reconstr Aesthet Surg 2009;62:955-63.

15. Takiwaki H, Shirai S, Kohno H, Soh H, Arase S. The degrees of UVB-induced erythema and pigmentation correlate linearly and are reduced in a parallel manner by topical anti-inflammatory agents. J Invest Dermatol 1994;103:642-6.

16. Wong Y, Lee SS, Goh CL. Hypopigmentation induced by frequent low-fluence, large-spot-size QS Nd:YAG laser treatments. Ann Dermatol 2015;27:751-5.
17. Kuroki T. Review of patients with acquired bilateral nevus of Ota-like macules. J Jpn Soc Aesthetic Plast Surg 1999;21:29-37.

18. Kunachak S, Leelaudomlipi P, Sirikulchayanonta V. QSwitched ruby laser therapy of acquired bilateral nevus of Ota-like macules. Dermatol Surg 1999;25:938-41.

19. Ee HL, Goh CL, Khoo LS, Chan ES, Ang P. Treatment of acquired bilateral nevus of ota-like macules (Hori's nevus) with a combination of the $532 \mathrm{~nm}$ Q-Switched Nd:YAG laser followed by the 1,064 nm Q-switched Nd:YAG is more effective: prospective study. Dermatol Surg 2006;32:34-40.

20. Wong THS. Picosecond laser treatment for acquired bilateral nevus of Ota-like macules. JAMA Dermatol 2018;154:1226-8.

21. Yu W, Zhu J, Yu W, Lyu D, Lin X, Zhang Z. A split-face, singleblinded, randomized controlled comparison of alexandrite 755-nm picosecond laser versus alexandrite 755-nm nanosecond laser in the treatment of acquired bilateral nevus of Ota-like macules. J Am Acad Dermatol 2018;79:479-86.

22. Wang BQ, Shen ZY, Fei Y, Li H, Liu JH, Xu H, et al. A population-based study of acquired bilateral nevus-of-Ota-like macules in Shanghai, China. J Invest Dermatol 2011;131:358-62.

23. Gupta GP, Gangwar DN. Naevus of Ota. Br J Ophthalmol 1965;49:364-8.

24. Wang HW, Liu YH, Zhang GK, Jin HZ, Zuo YG, Jiang GT, et al. Analysis of 602 Chinese cases of nevus of Ota and the treatment results treated by Q-switched alexandrite laser. Dermatol Surg 2007;33:455-60.

25. Watanabe S, Takahashi H. Treatment of nevus of Ota with the Q-switched ruby laser. N Engl J Med 1994;331:1745-50.

26. Alster TS, Williams CM. Treatment of nevus of Ota by the Qswitched alexandrite laser. Dermatol Surg 1995;21:592-6.

27. Liu J, Ma YP, Ma XG, Chen JZ, Sun Y, Xu HH, et al. A retrospective study of q-switched alexandrite laser in treating nevus of Ota. Dermatol Surg 2011;37:1480-5.

28. Kar HK, Gupta L. 1064 nm Q switched Nd: YAG laser treatment of nevus of Ota: an Indian open label prospective study of 50 patients. Indian J Dermatol Venereol Leprol 2011;77:56570.

29. Ueda S, Isoda M, Imayama S. Response of naevus of Ota to Qswitched ruby laser treatment according to lesion colour. Br J Dermatol 2000;142:77-83.

30. Chan HH, Leung RS, Ying SY, Lai CF, Kono T, Chua JK, et al. A retrospective analysis of complications in the treatment of nevus of Ota with the Q-switched alexandrite and Q-switched Nd:YAG lasers. Dermatol Surg 2000;26:1000-6.

31. Alexis AF, Sergay AB, Taylor SC. Common dermatologic disorders in skin of color: a comparative practice survey. Cutis 
2007;80:387-94.

32. Cardinali G, Kovacs D, Picardo M. Mechanisms underlying post-inflammatory hyperpigmentation: lessons from solar lentigo. Ann Dermatol Venereol 2012;139 Suppl 4:S148-52.

33. Anderson RR. Tattooing should be regulated. N Engl J Med 1992;326:207.

34. Brauer JA, Reddy KK, Anolik R, Weiss ET, Karen JK, Hale EK, et al. Successful and rapid treatment of blue and green tattoo pigment with a novel picosecond laser. Arch Dermatol 2012;148:820-3.

35. Kirby W, Desai A, Desai T, Kartono F, Geeta P. The KirbyDesai scale: a proposed scale to assess tattoo-removal treatments. J Clin Aesthet Dermatol 2009;2:32-7. 